\title{
Automatic Path Maker for Emergency Vehicles
}

\author{
A. Radha Rani, C.V.P.R. Prasad, D.B.K. Kamesh
}

\begin{abstract}
Automatic path maker for emergency vehicles" is the most convenient solution for the people. It is adequate condition into the traffic lights systems, as the emergency vehicles as route concerned in the higher priorities among the junctions as the road. At emergency vehicles as included into ambulances, rescue vehicles, fire brigades, polices, \& VIP persons. Which is many problem for then the problems, in while it is depended at the injury at the patients, personal accidents, fire fighting's, robbery, \& several vital situation. And can compulsory as implementing at techniques as to solve into situation.
\end{abstract}

The proposed system is for interrupt the running traffic signal when ambulance or VIP is near traffic signals lane. This RFID readed will send these messages to the traffic signal junction to release the news.

Keywords: Traffic control, Raspberry pi, RFID Tags, RFID Readers.

\section{INTRODUCTION}

Vehicle traffic controls as roads into crossing have been always at a matter on concerning at administration as several moderns city around in the worlds [4]. Among these apply into many attempts was make to designed into efficient automate system into solves in this problems. Moreover the present days system as used to pre determine time circuit to operates as traffic signal, when it is not very practically as becomes they are acting as before to the present congestions as the traffic into the crossing. Now, automate traffic controls system in that vehicle has waited as road crossings even among rather traffic into other directions. This is main problems are such as ambulance get into wedged up through a red traffic signals $\&$ wasted as valuable time, $\&$ can be lead as taken into risk as patient's life. In the normal, Congestion at translate and on lost time, missed as opportunity, lost worker productivity, delivery delay, $\&$ increased costs. The managed as non recurred into congestions, they will used to the raspberry pi. RFID tag as helping clearing to the heavy traffics. And Node MCU have been MQTT protocol with transmitting \& receiving with wireless connects into used to the Wi-Fi modules as used into provides as a network among reader \& raspberry pi. To solved in these problem \& an adding furthermore enhancement in the complex issues to vehicle traffic controls as road cross at an images recognition $\&$ send as information's nearby hospital \& vice versa. They will propose used to radio frequencies identifications (RFID). These are critical ideas among the principles of the RFID

Revised Manuscript Received on December 12, 2019

A.Radha Rani,Professor, Malla Reddy Engineering College for Women, Maisammguda, Hyderabad, TS

Dr. C.V.P.R. Prasad, Malla Reddy Engineering College for Women, Maisammguda, Hyderabad, TS

Dr. D.B.K.Kamesh, Malla Reddy Engineering College for Women, Maisammguda, Hyderabad, TS

tracks as vehicles. too, and can be worthly mentioned into the RFID tags, as design into explicits with scanned into the fast moving object (up to $15 \mathrm{cms}$ ), as a paper, developed into vehicle tracks as possibles. Presently, obtainable hardware may be uses into monitoring as a near distance upto $15 \mathrm{cms}$. The essentially as to known as the road traffics density into real time, particularly at metropolitan city, form signal controls \& effective managed in the traffic as emergency. One of the most common problems everywhere is traffic, although many control measures are applied. Still, there is a problem with traffic in emergency cases and ambulance vehicles. Many lives are under risk, due to heavy traffic and no proper traffic management

The proposed system is for interrupting the running traffic signal when an ambulance or VIP is near traffic signal lane, and the RFID reader will send to the traffic signal junction to release the message.

\section{A.Necessity:}

The adequate condition into the traffic light systems, the emergencies vehicles route concern to the higher priority among the junctions as the road. At the emergencies as vehicles as included ambulances, rescue vehicles, fire brigade, police, \& VIP persons. There are several problem for this problems, in which is dependent in the injury at the patients, personal accidents, fire fightings, robbery, \& several various vital situation. The compulsory is implemented into techniques as solved into this situations.

\section{B. Objectives:}

The proposed system is for interrupting the running traffic signal when ambulance or VIP is near traffic signal lane. The RFID reader will send the messages to the traffic signal junction to release the news.

In this generation, people prefer smart work only. The same goes for the cities. The term automation has led to a significant change in the world. Almost all countries artfully digitalized. In short, automation has become a relevant term, whether at home or outside the home. Our project focuses on traffic automation. The signals can be controlled manually from long distance as well.

To make the Smart environment that enables the user to monitor and control signals parameter on a real-time basis using RaspberryPi. To save the time of the emergency vehicles by using NodeMCU for network connection Taking into the considerations different advancements in the field of Electronics and Robotics, we can develop a method for monitoring signals by using RaspberryPi with the help of Internet of Things. It helps in the completion into making an automatic pathway more quickly in less period. 


\section{SYSTEM MODEL AND LITERATURE SURVEY}

\section{A.Literature Survey:}

“Automatic Path Maker for Emergency Vehicles "The project makes use of Raspberry Pi and RFID tags to control the signals using IoT based technology. "Automatic Path Maker for Emergency Vehicles"-The signals are controlled using Raspberry Pi, which Node MCU, which will be in the same network where it uses the MQTT protocol for transmitting and receiving information to the cloud server giving exact analysis. "Automatic Path Maker for Emergency Vehicles"- involves RFID tags and RFID Readers to detect the moving vehicles connected with RFID Readers. IoT based Industrial automation paper proposed by Sanyuta Swami and team.

Industrial Automation Uses as the Internet of Things (IoT) In these papers, there is developed as system is automatic monitored in the industrial application \& generated \& made into intelligent decision used to the concepts as iot. arduino and iot based industrial automation. Internet of things as completed through used to local networks standard \& remote controlling a\& monitoring signals parameter through RaspberryPi (IoT based Technology).The UK People have developed a series of small single-board computers. Raspberry $\mathrm{Pi}$ Foundation to encouragethe teaching of basic computer science in schools and developing countries.It does not take in peripherals and cases.

The originally is model because most populars than projects, as sell outside it is targets as marketing as used to like robotics. It is developed in the new technology as has been allows at moves with the1st Generation as the Internet to the current change over as the Fourth generations. IOT based the unique models because far more popularly in than expect, In this paper, Referred from IEEE paper of International Research Journal of Engineering and Technology Industrial Automation using IoT paper proposed by Bhosale. Then automatically creating an interface for controlling that appliance. The specification of each device includes a high-level description of every function, a hierarchical grouping of those functions, and dependency information, which relates the availability of each task to the appliance's state. Wireless Industrial Automation Using Raspberry Pi paper proposed by Thomas, Mathew and team.

\section{B.Block Diagram}

\section{RECEIVER}

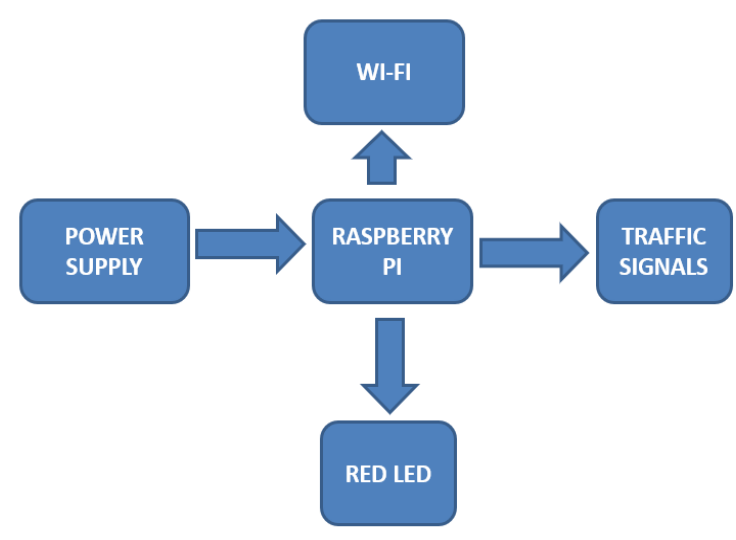

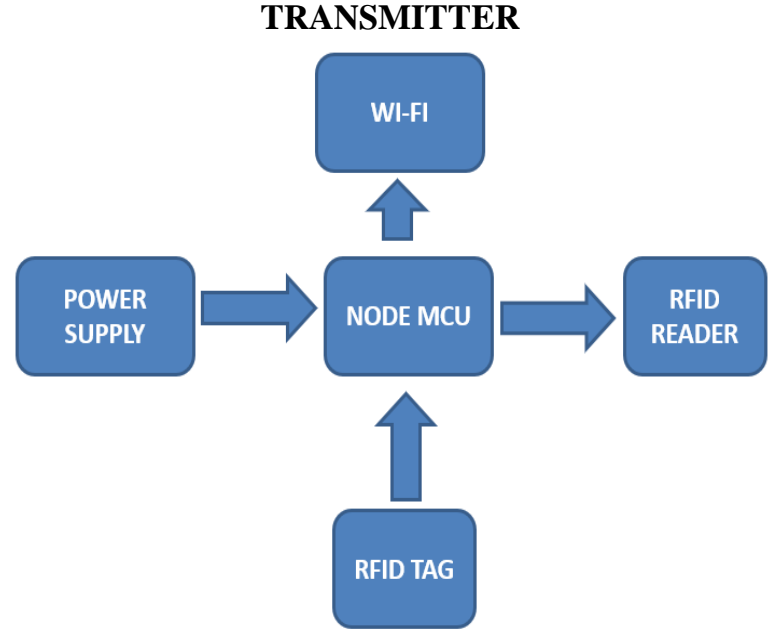

\section{a. Description of various blocks}

\section{WIFI:}

Wi-Fi networks physicallynot wired connection between the sender and receiver using radiofrequency (RF) technology.

\section{Node MCU:}

Node MCU is an open-source IoT platform that runs on the ESP8266 Wi-Fi SoC from Express the firmware based on an ESP-12 module. The term "NodeMCU" are pure development kit as rather than the firmwares. Between applyi Lua scripting languages into firmware. It is base as the eLua projects \& built into the Express if Non OS SDK with ESP8266. Among the used to several open source project. Likelua-cjson \& SPIFFS.

\section{RFID Reader:}

Radio-frequencyidentification (RFID) is used to electromagnetic field to identify \& tracks tag attach into object automatic. The card is contains into electronics as stores information's. Passive tag gathering as energy at a hard through RFID reader crossed examine as radio wave. Energetic tag has been locally power source like at battery \& can operates as hundred into meter into the RFID readers. Uncertain an barcodes, in the cards require is not with the line of sight as the reader therefore that it can be root into the track objects. RFID as one methods to automatic identifications \& data captures (AIDC).

\section{RFID Tag:}

The Radio Frequencies Identifications Tag (RFID) to an electronic tags in that conversation data's for as RFID read into radio waves.

Most of RFID tags is finish as at least two part. The antenna to the first one that receive as radio frequencies (RF) wave. The another one as an integrated circuit (IC), while it is uses as processing \& store data's, as known modulated \& demodulated in the radio wave receive or sent through the antenna. 


\section{HARDWARE DESCRIPTION}

\section{A. Introduction}

Designed as called as the first stepped into famousin the implement as phases form any technique \& principle to the defined as a devices, on process \& system into satisfactions detailed as to certifications in its physically awareness.

One of the software requirement has been examine \& specifies, in the software designs as involved as three technical activity designed, coding, implementations, \& testing as carry out.

The designing into activity is the primary important in the phases so, into activities, decision ultimate affects in the software implementations \& maintenance is done. The decision has been the finally as bear upon the reliable \& maintain as the systems. Designed as the one way to translated the customer requirement into date accurate.

\section{B. Circuit diagram}

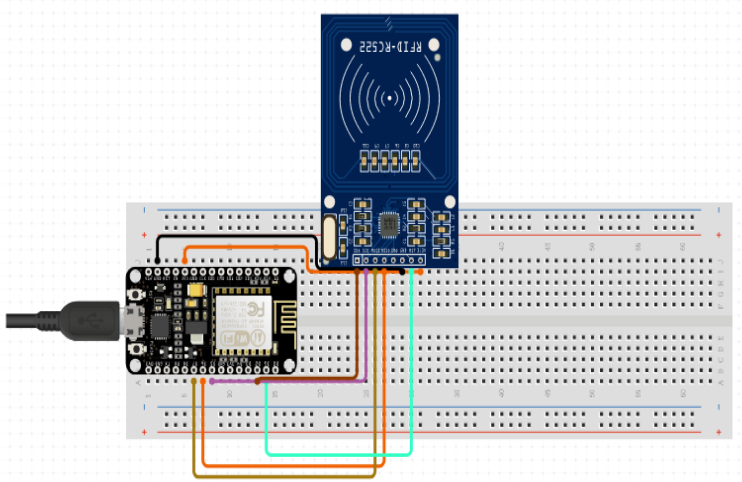

NodeMCU connected to the RFID reader

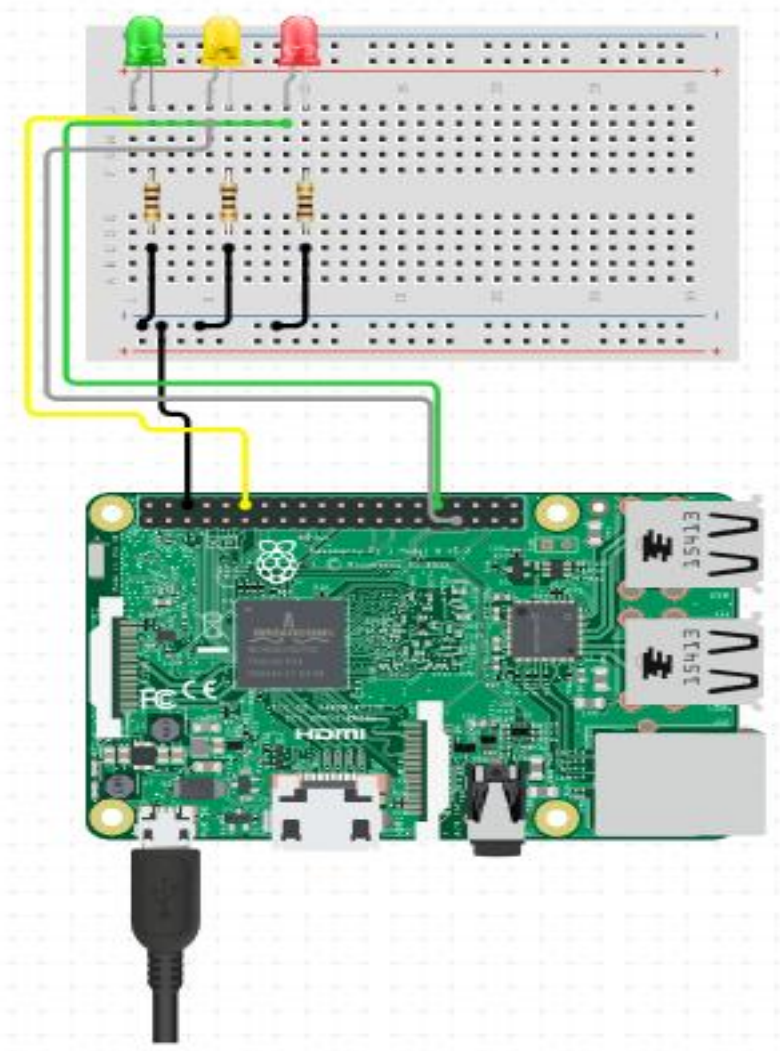

RFID connections

\section{C.Description of components}

Raspberry pi:

Raspberry Pi is trivialin size computers mainly designed for children to improve their knowledge over the extensive network. Raspberry Pi is built on an ARM processor and runs off the Linux platform, not precisely the Linux but a distribution of Linux. Raspberry Pi consists of 512 MB ram with two USBs also with an Ethernet cable connector. The Raspberry pi can makeit to a TV or an HDMI monitor through HDMI cable. It also supports analog and HDMI audio. Now let's list down what are the required accessories for Raspberry Pi.

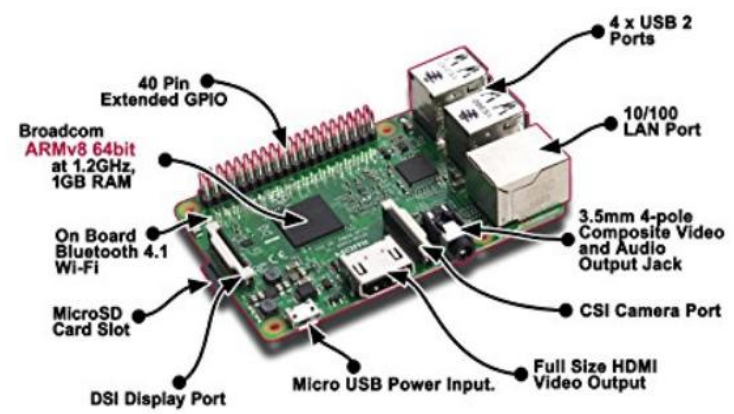

Looking at the circuit board, you can see:

- The Processor and RAM chip

- The LAN controller chip

- An HDMI video output connector

- A composite analog video connector (on models A and B)

- An SD card connector

- A micro-USB power connector

- A USB port

- An Ethernet port (on model Bs)

- A camera connector

- And the essential GPIO headers

\section{Technical features of the Raspberry pi}

- SoC: Broadcom BCM2837 (roughly 50\% faster than the Pi 2)

- CPU: $1.2 \mathrm{GHz}$ quad-core ARM Cortex A53 (ARMv8 Instruction Set)

- GPU: Broadcom Video Core IV@400 MHz

- Memory: 1 GB LPDDR2-900 SDRAM

- USB ports: 4

- Network: 10/100 MBPS Ethemet, 802.11n Wireless LAN, Bluetooth 4.0

\section{Making a bootable SD card for Pi:}

Now let's go ahead with building a bootable SD card for Pi. Step1: Download the image of a Linux distribution NOTE: The Windows platform is shown below

First, download the picture of a Linux distribution from the official site of Raspberry Pi (raspberrypi.org). In downloads, you can find the distributions of Linux. 


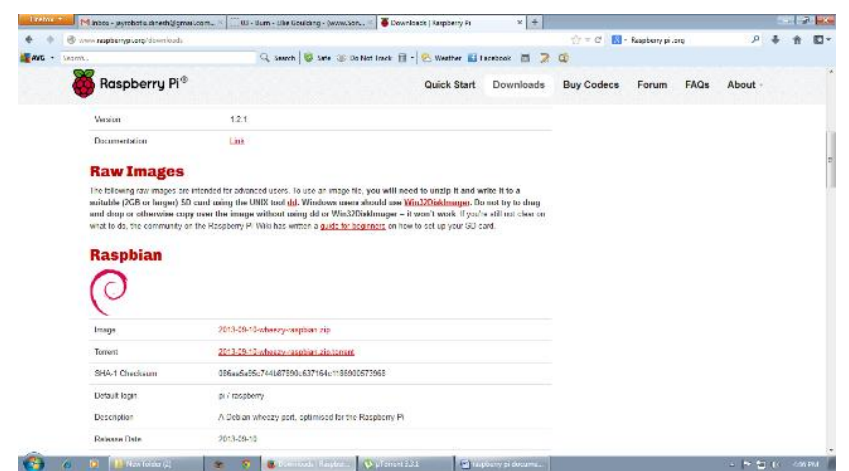

Download Raspbian from the page and save it on your computer

Step2: Download win32diskimager

Win32 disk imager is used to burn the downloaded OS image into the SD card. Download the software, run it, and follow the steps:

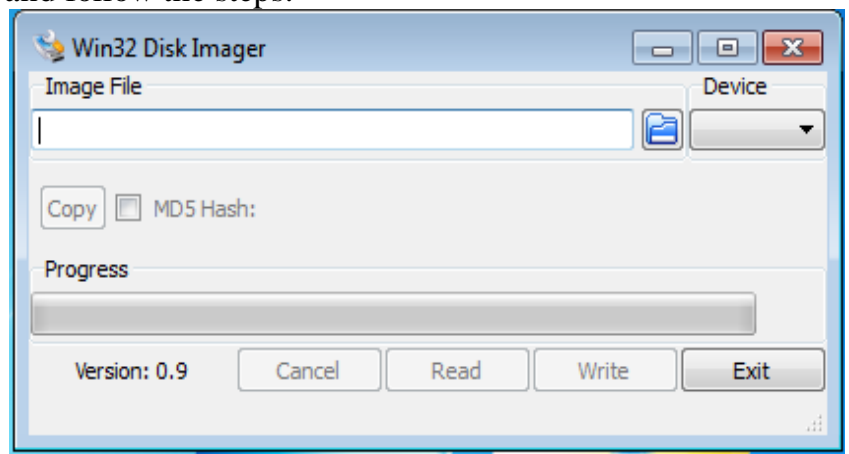

Browse the image file of OS downloaded in step1 from your computer and click on the write button. It burns the image file onto the SD card.

Other ways of making a bootable SD card:

\section{Using New Out of Box (NOOBS):}

Download it from raspberrypi.org site. It will download a zip file containing some files. Don't forget to download the formatting tools because first, you should format the SD card with the formatting tools and copy the extracted data of NOOBS into the formatted SD card.

Pi Recovery - BuiltsMay 242013

Now when you power ON Raspberry Pi with the SD card inserted into it, you will come across the above screen from which you can select an OS you want and click on install OS. The OS will automatically get installed, and Raspberry Pi will start booting with that OS.

Using berry boot:Berry boot is software that is used to install the selected OS from the network and install the selected OS on Raspberry Pi.

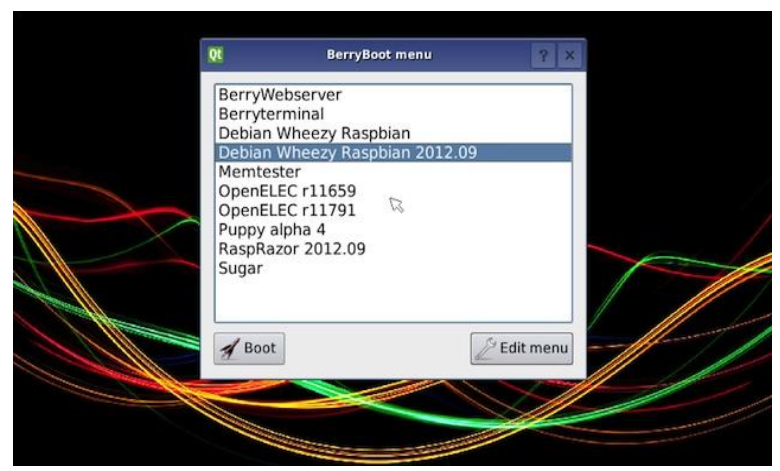

Select the OS you want and click on BOOT. That's it.

Now you got an OS on the SD card, and the Raspberry Pi can boot with that SD card. Now when you start booting the Raspberry Pi for the first time, it asks you for some configuration setting for the first time.

\section{Configuring Raspberry Pi:}

When you boot your Raspberry Pi for the first time, you will come across the following screen for some configuration settings.

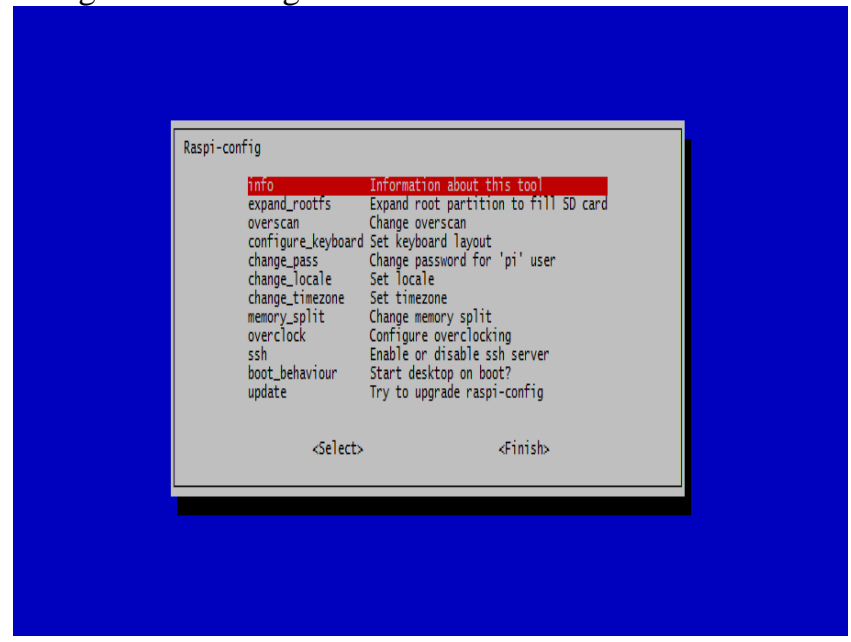

\section{Overscan:}

The default overscan option is disabled. Ifyou enable the overscan and alteration the values to fit the image to the screen. The values show the amount of overscan so that the display software can correct; use positive values if the picture goes off the screen, harmful if there are black borders around the edge of the display.
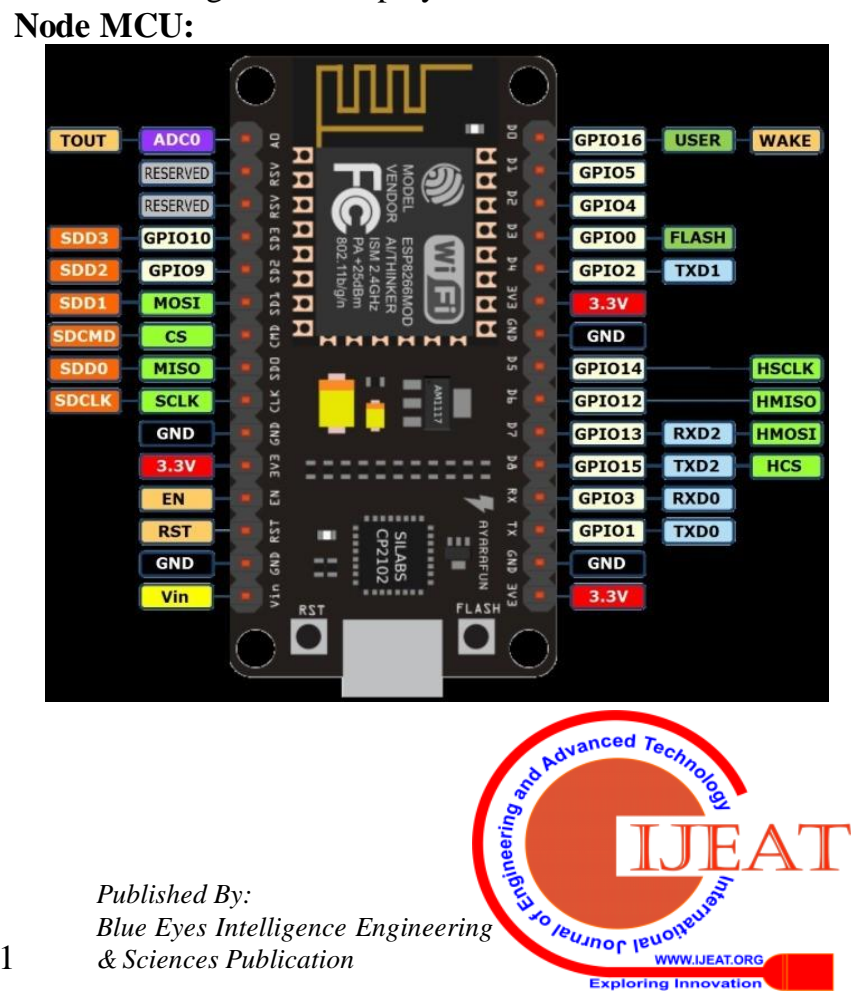


\section{What Makes It Special}

In various feature into Arduino controller as made as stand out within the rests as the micro controller in the markets. In this examples, in the software as opened as sources, therefore we can be "looking as under in the covering," Because speaks, \& it is free as downloading, modification, \& re use in when it always an plus with more advance developer. Even if the requires as plan with the Arduino is open sources, therefore user may builds in their owns into have to buying once. Many company to make the Arduino board, too, \& you may be buying to full kit breadboard, wire sensor, \& more. Both fullyassembled, Arduino as the low ending to the costs spectrum \& as compatible for Window, Mac, \& Unix system.

The project is to made micro controller is accessible on everybody, both these are minimal computers backgrounds, regardless as the age.

It is different techniques as Arduino boards, together.

\section{What You Need:}

Working to the NODEMCU, They needed as a laptops, desktops, and tablets than your may downloading the Arduino developmental into environments. It has been write into Java \& can be colding at Window, Mac OS X 10.7 Lion as newer, or Linux 32 and 64 bits. The connected as ayour board for your computers, your can be needed as a USB data cables. Unlike USB charges and synching wire, they can be squares, block shape interfaces as instead into small interface such as the micro USB. Unless at a well establishing into electronics hobby for ton of the cold sensor \& like lying around, we invested as a kits. Form tests \& prototypes, your laptop provide in the power form the Arduino by means of the USB data cables. Therefore, form independent laptop project, and it can be probable need to given as independents, portable powersource with your projects. At links into provide under online resource in that shows how to uses as battery power with the Arduino.

\section{IMPLEMENTATION AND DEBUGGING}

\section{A.Hardware Development}

In this project, Raspberry Pi Python language and Node MCU is programmed in embedded $\mathrm{C}$ language and used to turn on and off the signals, connect through RFID tags and RFID readers. The Raspberry pi connected to Node MCU such a way that it involves in controlling and monitoring the messages, and the received analysis data will be displayed in the cloud server

The following connection of the components does the above execution:

1. WIRED AND WIRELESS CONNECTIONS:

WIRED: 1 . RFID Reader to Node cu

2. Raspberry pi to 3 sets of signals

WIRELESS: Node MCU, Raspberry pi connected to a shared network

2. RFID Reader connected to the Wi-Fi module.

The Raspberry Pi connected via PUTTY or Remote Desktop.

\section{B.Software development}

\section{a. Flow chart}

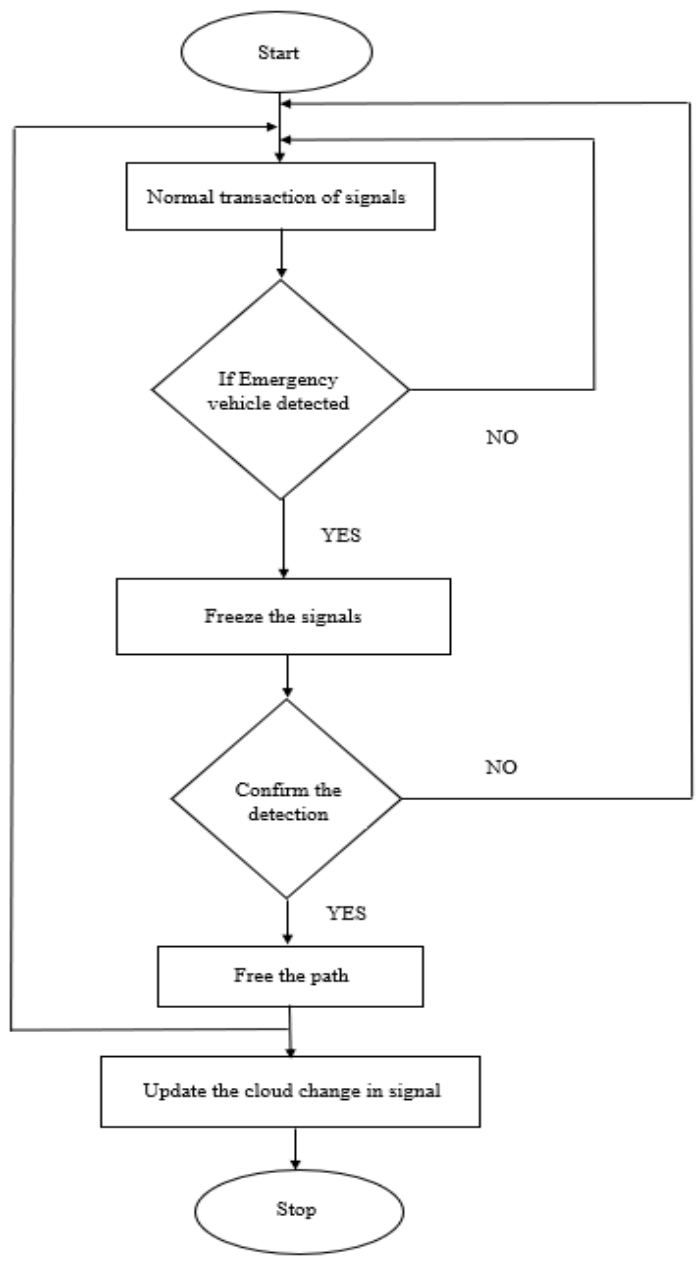

Main Program Code

\section{C.Interfacing of hardware:}

1. Give power connection to Raspberry Pi and Node MCU.

2. You will find the transactions of signals in the project.

3. Check whether the signals are detected with the help of RFID Readers and RFID tags.

4. This is how the whole project is interfaced to our cloud server.

5. All the signals are connected to the raspberry pi, which is mainly linked to this Node MCU (having MQTT Protocol), which is IoT based

\section{D.Debugging and testing of hardware and software:}

Connection establishment between the Node-MCU, and Raspberry pi. Verifying the power supply and the voltage that is being passed by the Raspberry pi. We need to check that there must be one secured network for connecting the modules present in the project. Readings from the RFID reader must be sent thoroughly to the Raspberry pi via Node-MCU. This can be done by opening the running code in any PC by clicking the serial monitor for Node-MCU delivery, and for raspberry pi delivery, we need to run the raspberry pi code in the pi either by using the remote desktop connection or by using putty application. Check the signal transition for various RFID readings and later to that. 


\section{Automatic Path Maker for Emergency Vehicles}

\section{E.Final words on working of the project:}

Technology is meant to provide a secure life for all living beings. According to that rule, our plan is majorly for providing security to all the experiences. With the unaltered access to the modules and signals, we can provide quick and fast transitions of messages without any human interference. For monitoring of the words, we are offering cloud updates depending on the date and time for the access of superiors of the government.

\section{F. Output Screens and Project Picture:}
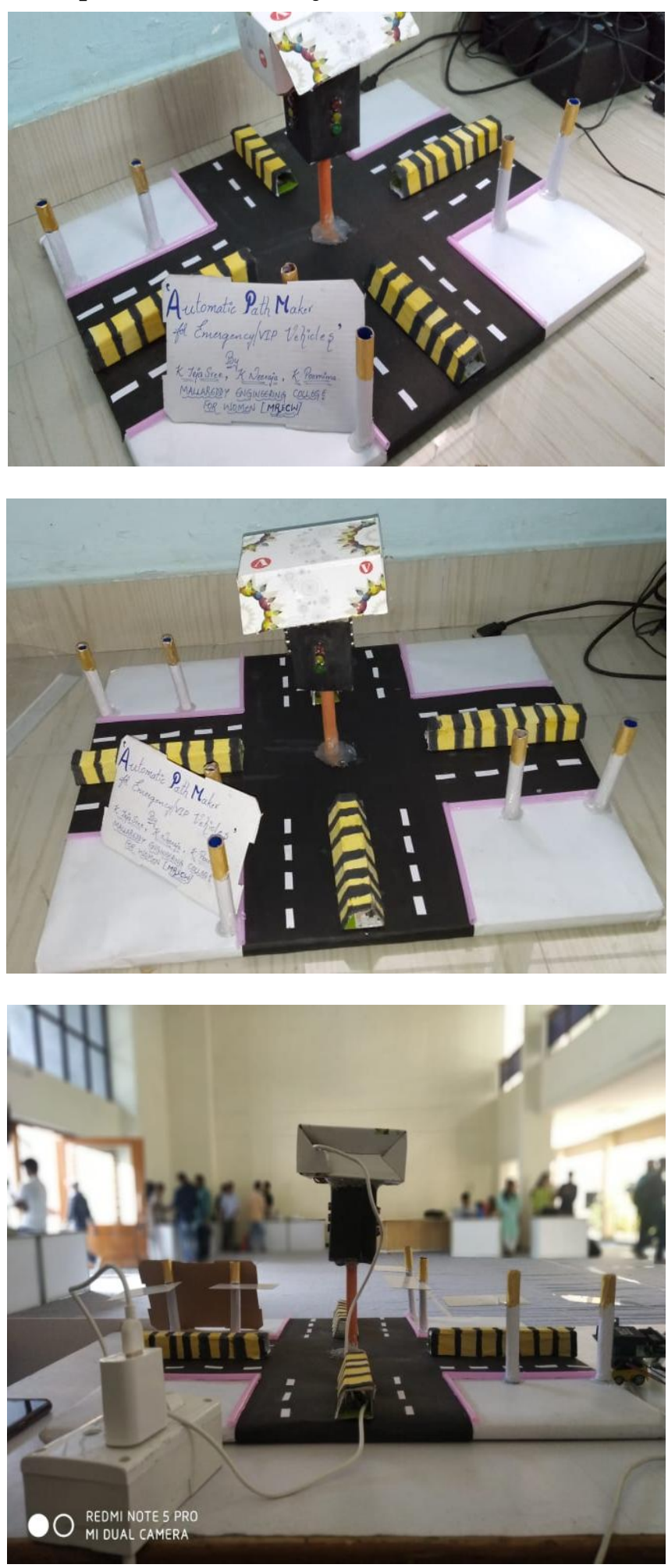

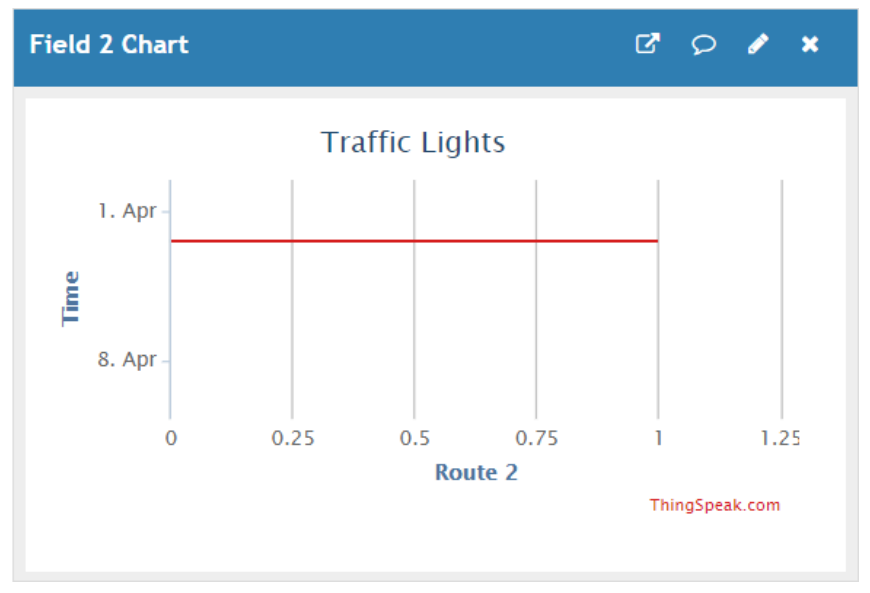

Field 3 Chart $\quad$ ए $0, x$

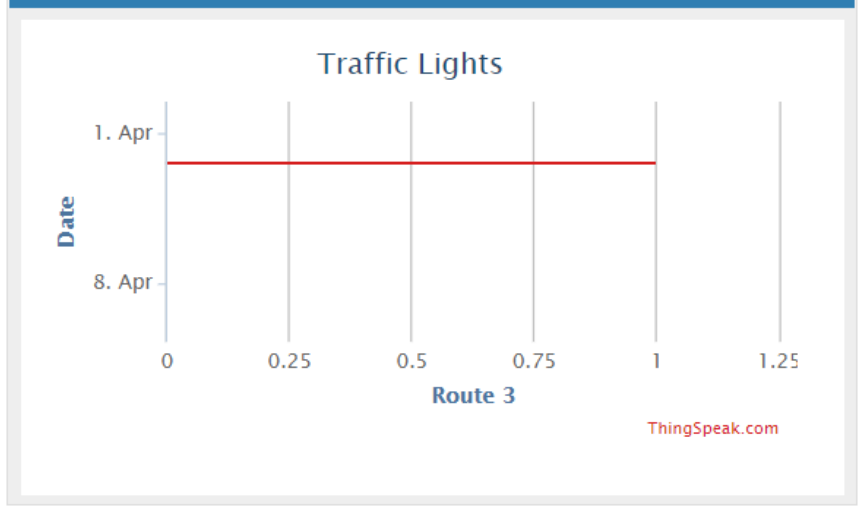

\section{CONCLUSION AND FUTURE SCOPE}

\section{A.Conclusion:}

This paper is beneficial in places where there are heavy traffic and busy areas. It helps in auto transmitting the signal junctions without human resources. As this is wireless technology, we can further improve the accuracy with real-time implementation.

\section{B.Future scope:}

This project can be further enhanced with IoT technology to achieve high performance in current technology. We can also develop an android application for the user to make it a more natural operation side part. 


\section{REFERENCES}

1. Dinesh Mohan, Omer Tsimhoni, Michael Sivak, Michael J Flannagan Road safety in India: challenges and opportunities -Repost numberUMTRI-2009-1

2. Manisha Ruikar, National statistics of road traffic accidents in India, Journal of Orthopaedics, Traumatology and Rehabilitation, 23 Sep. 2013, vol. 6, issue 1, pp. 1-6

3. Daniel Brookoff, Charles S. Cook, Charles Williams, and Calvin S. Mann, Testing Reckless Drivers for Cocaine and Marijuana, The New England Journal of Medicine, Aug. 25, 1994, pp. 518-522.

4. V.B.Nitya, N.SrideviArun \& K.Pujari,Linear octree by volume intersection using perspective silhouettes, Pattern Recognition Letters, ISSN: 0167-8655, Vol: 13, Issue: 11, Page: 78

\section{AUTHORS PROFILE}

A.Radha Rani,Professor, Malla Reddy Engineering College for Women, Maisammguda, Hyderabad, TS

Dr. C.V.P.R. Prasad, Malla Reddy Engineering College for Women, Maisammguda, Hyderabad, TS

Dr. D.B.K.Kamesh, Malla Reddy Engineering College for Women, Maisammguda, Hyderabad, TS 